\title{
A COUPLING METHODOLOGY TO MODEL NEAR AND FAR FIELD EFFECTS OF STRUCTURES AND ENERGY DEVICES DUE TO WAVE INTERACTION
}

\author{
Vasiliki Stratigaki, Ghent University, Vasiliki.Stratigaki@UGent.be \\ Peter Troch, Ghent University, Peter.Troch@UGent.be \\ David Forehand, The University of Edinburgh, D.Forehand@ed.ec.uk
}

\begin{abstract}
INTRODUCTION
This study focuses on the numerical modeling of wave fields around structures due to their interaction with waves, with the intention to simulate both the resulting near and far field effects. Examples from the wave energy world are employed such as Wave Energy Converters (WECs), fixed or oscillating devices usually arranged in farms, that interact with the incoming waves and extract wave energy from them. As a result of the hydrodynamic interaction between the devices within a farm (so-called near-field effects), the power absorption of the farm is affected. Moreover, wave dissipation has been observed numerically (e.g. Troch et al., 2010) and in scale tests (e.g. Stratigaki et al., 2014; 2015) between the WEC farm location and e.g. the shoreline (socalled far-field effects). These wave field changes can affect neighboring sea activities, coastal eco-systems, the coastline and even coastal defense conditions/parameters.
\end{abstract}

\section{PROBLEM STATEMENT}

As a result of the interaction between WECs and the incident wave field, additional wave fields are generated: the radiated (for oscillating WEC types) and the diffracted wave field around each WEC, which together with the incident wave field compose the total (or 'perturbed') wave field around the WECs. Several numerical methods are employed to analyse these wave fields: (a) To simulate physically correct near field effects, wave-structure (wave-WEC) interactions and wave energy absorption, the approach of models based on Boundary Element Methods (BEM) for solving the potential flow formulation or models based on the Navier-Stokes equations (CFD models) is employed; (b) To investigate far field effects of WEC farms in large areas, the approach of wave propagation models is the most suitable.

However, all these models suffer from a common problem; they cannot be used to model simultaneously near and far field effects due to limitations (Troch \& Stratigaki, 2016): (1) wave-structure interaction solvers suffer from a high computational cost, when simulating power absorption and the wave field alteration due to large WEC farms and are used typically for less than 10 WECs; (2) wave propagation models enable simulation of far field effects. Large WEC farms installed in large domains are modeled at a reasonable computational cost. As a result, the changes in wave field and the associated environmental impacts on the sea and the shoreline can be studied at regional scale. However, the WECs are approximated up to now by using parameterized energy sinks and empirically tuned energy absorption coefficients, and therefore this method only partially addresses the underlying physics.

\section{COUPLING METHODOLOGY AND DISCUSSION}

A generic coupling methodology is presented here (Fig. 1), developed to combine the previously described two approaches ( $a$ and $b$ ). In addition, a new wave generation technique is presented, for generating the total wave field induced by a WEC in a wave propagation model. A wave generation circle is used around the WEC, on which prescribed internal boundary wave conditions are imposed, provided by a wave-structure interaction solver (Fig. 2).

Both, the proposed coupling methodology and wave generation technique are generic: (i) the coupling can be realized between any wave-structure solver and wave propagation model, (ii) they apply to any (oscillating/floating) structure, including oscillating water columns and all WEC types, (floating) breakwaters, platforms, etc. For the validation of the proposed coupling methodology, a test case using a heaving WEC is used, for which coupling between the wave-structure interaction solver, WAMIT (www.wamit.com), and the time domain wave propagation model, MILDwave (Troch, 1998), has been realized. The results obtained for the diffracted, radiated and total wave field around the WEC under incident waves using the coupling methodology are validated against the results obtained from the wave-structure model, showing very good agreement. Finally, the benefits of the proposed coupling methodology to model floating bodies in a phase resolving wave propagation model are discussed.

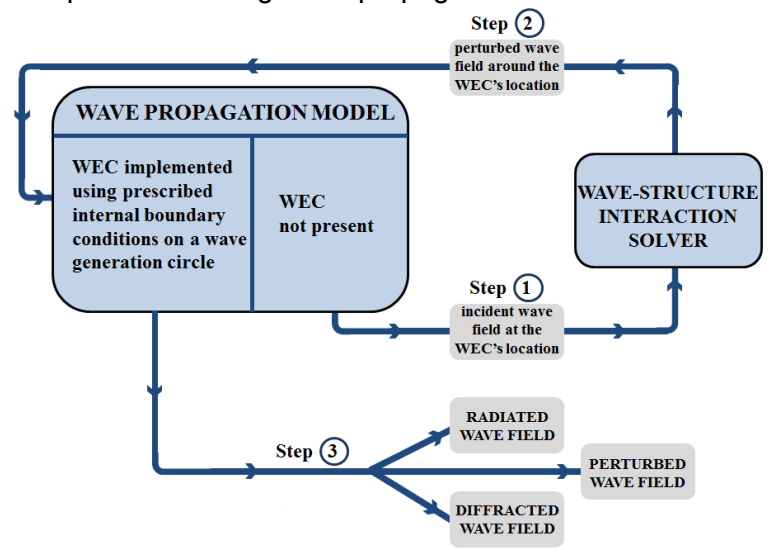

Figure 1 - Flow chart of the step-by-step procedure for realizing the proposed generic coupling methodology between the approach of a wave-structure interaction solver and a wave propagation model, respectively, to predict near and far field effects of a single WEC or a WEC farm.

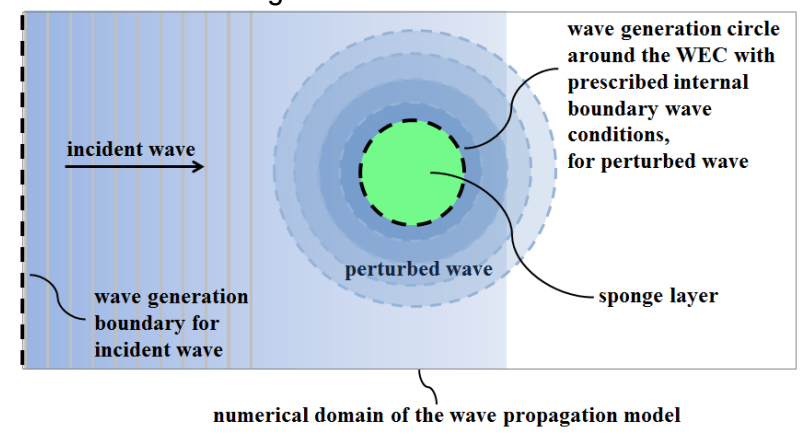

Figure 2 - Definition sketch of the technique of the wave generation on a circle around the WEC (or WEC farm) using prescribed internal boundary wave conditions for the total wave field around the WEC (farm). This total wave field is derived from a wave-structure interaction solver.

\section{REFERENCES}

Troch \& Stratigaki (2016): Phase-Resolving Wave Propagation Models. In: Folley (Ed), Numerical Modelling of Wave Energy Converters, Elsevier, Ch. 10, 27 pp. ${ }^{*}$ Stratigaki, Troch, et al. (2014): Wave basin experiments with large wave energy converter arrays to study interactions between the converters \& effects on other users in the sea and the coastal area. Energies, 7, 701-734. * Stratigaki, Troch. et al. (2015): Sea-state modification \& heaving float interaction factors from physical modelling of arrays of wave energy converters. J. Renew. Sustain. Ener. 7. * Troch (1998): MILDwave - A numerical model for propagation \& transformation of linear water waves. Int.Report, Ghent University. * Troch, et al. (2010): Wake effects behind a farm of wave energy converters for irregular long-crested \& shortcrested waves.ICCE. * WAMIT, http://www.wamit.com/manual.htm. 